\title{
Value of Candida Polymerase Chain Reaction and Vaginal Cytokine Analysis for the Differential Diagnosis of Women with Recurrent Vulvovaginitis
}

\author{
Stephanie Weissenbacher, ${ }^{1}$ Steven S. Witkin, ${ }^{2 *}$ Vera Tolbert, ${ }^{2}$ \\ Paulo Giraldo, ${ }^{2,3}$ Iara Linhares, ${ }^{4}$ Andrea Haas, ${ }^{1}$ \\ E. Rainer Weissenbacher, ${ }^{1}$ and William J. Ledger ${ }^{2}$ \\ ${ }^{1}$ Departments of Obstetrics and Gynecology, Klinikum Grosshadern, University of Munich Medical \\ School, Munich, Germany \\ ${ }^{2}$ Weill Medical College of Cornell University, New York, NY \\ ${ }^{3}$ University of Campinas Medical School, Sao Paulo, Brazil \\ ${ }^{4}$ Hospital das Clinicas, University of Sao Paulo Medical School, Sao Paulo, Brasil
}

\begin{abstract}
Objectives: Recurrent vulvovaginitis remains difficult to diagnose accurately and to treat. The present investigation evaluated the utility of testing vaginal specimens from women with symptomatic recurrent vulvovaginitis for Candida species by polymerase chain reaction (PCR) and for cytokine responses.

Methods: Sixty-one consecutive symptomatic women with pruritus, erythema, and/or a thick white discharge and a history of recurrent vulvovaginitis and 31 asymptomatic women with no such history were studied. Vaginal swabs were tested for Candida species by PCR, for the antiinflammatory cytokine interleukin (IL)-10, and for the proinflammatory cytokine IL-12.

Results: C. albicans was detected in $19(31.1 \%)$ of the patients as well as in three $(9.7 \%)$ controls $(P=0.03)$. Both IL-10 $(31.1 \%$ vs. $0 \%)$ and IL-12 $(42.6 \%$ vs. $6.5 \%)$ were also more prevalent in the recurrent vulvovaginitis patients $(P<0.001)$. However, there was no relation between the presence or absence of Candida and either cytokine. Detection of IL-12 in 14 women indicated the stimulation of a vaginal cell-mediated immune response possibly from an infectious agent. The presence of only IL-10 in six patients indicated a suppression of vaginal cell-mediated immunity and was consistent with a possible allergic etiology. The absence of both IL-10 and IL-12 in other patients, similar to that found in healthy controls, suggested a noninfectious, nonallergic etiology of their symptoms.

Conclusion: Many women with recurrent vulvovaginitis are not infected with Candida. Testing for Candida should be required in this population. Treatment with only anti-Candida medication will clearly be inadequate for the majority of women with this condition. Infect. Dis. Obstet. Gynecol. 8:244-247, 2000. @ 2000 Wiley-Liss, Inc.
\end{abstract}

$\mathrm{R}$ ecurrent vulvovaginitis remains a difficult disorder to diagnose accurately, to treat, and for which to end the repetitive cycles. There is a widespread assumption that a Candida infection is al- ways, or nearly always, involved in eliciting the associated symptoms. The sale of nonprescription drugs for self-treatment of vaginal "yeast" infections is many times greater than the number of

\footnotetext{
These studies are part of the doctoral thesis requirement for Stephanie Weissenbacher at the Ludwig Maximilians University, Munich, Germany.

*Correspondence to: Dr. Steven S. Witkin, Department of Obstetrics and Gynecology, Weill Medical College of Cornell University, 515 East 71st Street, New York, NY 10021. E-mail: switkin@mail.med.cornell.edu
} 
infected women. Many clinicans also treat on the basis of clinical symptoms, without confirmation that Candida is indeed present. This may account, in part, for the ineffectiveness of antifungal treatments in many women.

There is an increasing awareness that a localized immune perturbation may lead to vulvovaginitis in some women. A localized allergic response in the vagina has been demonstrated. ${ }^{1-4}$ Vaginal symptoms may be due to an immediate hypersensitivity response to infectious agents or to noninfectious allergens $^{1-3}$ as well as to eosinophil accumulation. ${ }^{4}$ The subsequent production of prostaglandin $E_{2}$ and inhibition of cell-mediated immune responses $^{1,5}$ would also favor proliferation of Candida or other microorganisms to levels capable of eliciting clinical symptoms.

To obtain a more accurate diagnosis of women with recurrent vulvovaginitis as the basis for selective treatment, we analyzed vaginal specimens from women with this disorder for Candida species by polymerase chain reaction (PCR) and for vaginal concentrations of interleukin (IL)-12 and IL-10. Induction of specific immune responses is largely dependent on IL-12. Only those T lymphocytes belonging to the Th1 subset express the $\beta$ chain of the IL-12 receptor. Binding of IL-12 to this receptor leads to Th1 lymphocyte activation and induction of a cell-mediated immune response. ${ }^{6}$ This is the mechanism responsible for limiting Candida growth in the vagina. ${ }^{1}$ However, in the absence of IL-12 $\beta$-receptor expression, the $T$ lymphocytes develop along the Th2 pathway. This leads to IL10 release, production of $\mathrm{IgE}$, induction of an allergic response, and inhibition of cell-mediated immunity. ${ }^{7}$ Under these conditions, vaginal Candida yeast can multiply and begin to germinate into their hyphal form. ${ }^{8}$

\section{MATERIALS AND METHODS Subjects}

The study population consisted of 61 consecutive women with at least four previous episodes of symptomatic vulvovaginitis in the past 12 months and who were currently complaining of pruritis, erythema, and/or a vaginal discharge. Thirty-one consecutive asymptomatic women seen at the same center with no self-reported history of vulvovaginitis and no evidence of a current vaginal infection or inflammation were also studied. All subjects were white. Women with herpesvirus, human papillomavirus, and bacterial vaginosis as defined by clinical criteria were excluded. All subjects were tested for Mycoplasma hominis, Ureaplasma urealyticum, Chlamydia trachomatis, Neisseria gonorrhoeae, syphilis, and HIV. None was receiving medication at the time of the study and for at least 4 weeks previously. Informed consent was obtained from each participant.

\section{Specimen Collection}

A vaginal specimen was obtained by instilling $1.0 \mathrm{ml}$ phosphate-buffered saline (PBS) into the posterior vagina, rubbing the lateral walls with a cotton swab, and removing the specimen with a syringe. The sample was centrifuged, and the supernatant and pellet were frozen separately at $-80^{\circ} \mathrm{C}$ until tested.

\section{Candida Testing}

The pellet fractions were thawed, treated with lyticase to disrupt the Candida cell wall, and tested for $C$. albicans by PCR. ${ }^{9}$ This assay has previously been demonstrated to detect all $C$. albicans culturepositive specimens from the vagina as well as in additional culture-negative specimens. ${ }^{10,11}$ Negative specimens were retested by another PCR that is able to detect all other Candida species. ${ }^{12}$

\section{Cytokine Analysis}

The supernatant fractions were thawed and assayed for IL-10 and IL-12 by commercial ELISA assays (BioSource, Camarillo, CA). Values were converted to picograms per milliliter by reference to a standard curve generated in parallel to the test specimens. The lower limits of sensitivity were 5 $\mathrm{pg} / \mathrm{ml}$ for IL-10 and $1 \mathrm{pg} / \mathrm{ml}$ for IL-12. The positive values ranged from 10 to $144 \mathrm{pg} / \mathrm{ml}$ for IL-10 and from 31 to $164 \mathrm{pg} / \mathrm{ml}$ for IL-12. Duplicate values differing by more than $10 \%$ were reassayed.

\section{Statistical Analysis}

Differences between variables were evaluated by Fisher's exact test and the nonparametric Mann-Whitney test, as appropriate. A $P$ value $<0.05$ was considered significant.

\section{RESULTS \\ Demographic and Historical Variables}

A comparison of the patient and control populations is shown in Table 1 . There were no statisti- 
TABLE I. Demographic and historical variables in patients and controls

\begin{tabular}{lcc}
\hline Variable & $\begin{array}{c}\text { Patients } \\
(\mathrm{n}=61)\end{array}$ & $\begin{array}{c}\text { Controls } \\
(\mathrm{n}=31)\end{array}$ \\
\hline Mean age (S.D.) in years & $31.6(7.1)$ & $34.4(6.2)$ \\
Mean no. pregnancies (S.D.) & $1.1(1.2)$ & $1.1(1.0)$ \\
Allergic history ${ }^{\mathrm{a}}$ (\%) & 43.7 & 32.3 \\
Current smoker (\%) $_{\text {Oral contraceptive user (\%) }}$ & 16.3 & 25.8 \\
\hline
\end{tabular}

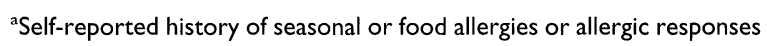
to medications.

cally significant differences in any of the variables examined. Except for detection of $M$. hominis and/ or $U$. urealyticum in seven of the patients, none of the patients or controls was currently positive for any of the non-Candida microorganisms examined. The presence of these mycoplasmas appeared to be unrelated to the cytokine findings.

\section{Detection of Vaginal Candida Species and Vaginal Cytokines}

C. albicans was detected in the vaginal specimens from only $19(31.1 \%)$ of the women with symptomatic recurrent vulvovaginitis. Three $(9.7 \%)$ of the healthy controls were also positive for this organism $(P=0.03)$. No additional Candida species were detected in any of the specimens.

IL-12 was present in $42.6 \%$ of the vaginal samples from symptomatic women with recurrent vulvovaginitis, and $31.1 \%$ were positive for IL-10. In contrast, only two $(6.5 \%)$ control vaginal specimen contained IL-12, and none was positive for IL-10 $(P<0.001)$. There was no relation between the presence or absence of Candida in the recurrent vulvovaginitis patients and any of the variables examined (Table 2).

The relationships between cytokine and Candida detection in the recurrent vulvovaginitis patients are detailed in Table 3. In almost one-third of the symptomatic patients, neither Candida nor IL-12 or IL-10 was detected. A mixture of IL-12 and IL-10 was present in 14 women, 12 had only IL-12, and 6 were positive only for IL-10 in their vaginas. In nine women, Candida was detected in the absence of either cytokine.

\section{DISCUSSION}

By utilizing the very sensitive PCR, C. albicans was identified in only $31.1 \%$ of symptomatic
TABLE 2. Relation between $C$. albicans and various parameters in women with symptomatic recurrent vulvovaginitis

\begin{tabular}{lcc}
\hline & $\begin{array}{c}\text { Candida } \\
\text { present } \\
(\mathrm{n}=19)\end{array}$ & $\begin{array}{c}\text { Candida } \\
\text { absent } \\
(\mathrm{n}=42)\end{array}$ \\
\hline Variable & 47.4 & 38.6 \\
Vaginal interleukin-12 (\%) & 31.6 & 31.8 \\
Allergic history (\%) & 31.6 & 36.4 \\
Current smoker (\%) & 10.5 & 9.1 \\
Mean No. pregnancies (S.D.) & $1.4(1.2)$ & $1.1(1.3)$ \\
Mean age (S.D.) in years & $33.2(7.2)$ & $31.0(7.4)$ \\
Mean no. episodes of vaginitis per & & \\
$\quad$ year (S.D.) & $8.7(2.5)$ & $8.2(7.4)$ \\
Oral contraceptive user (\%) & 31.5 & 55.8 \\
\hline
\end{tabular}

TABLE 3. Detection of IL-10, IL-12, and C. albicans in vaginal specimens from women during an episode of recurrent vulvovaginitis

\begin{tabular}{llc}
\hline Cytokines & Candida & No. patients (\%) \\
\hline Only IL-10 & Present & I (I.6) \\
Only IL-10 & Absent & $5(8.2)$ \\
Only IL-12 & Present & $4(6.6)$ \\
Only IL-12 & Absent & $8(13.1)$ \\
IL-12, IL-10 & Present & $5(8.2)$ \\
IL-12, IL-10 & Absent & $9(14.8)$ \\
None & Present & $9(14.8)$ \\
None & Absent & $20(32.8)$ \\
\hline
\end{tabular}

women with recurrent vulvovaginitis; none was positive for other Candida species. Therefore, the vaginal symptoms in the majority of these patients were not due to the presence of Candida at this anatomical site. In addition, there was no consistent pattern of vaginal cytokine production in these women.

The heterogeneity of cytokine responses indicates multiple etiologies of the clinical symptoms. The presence of IL-12 in 26 of the patients indicated a localized cell-mediated immune activation, consistent with a possible infectious etiology for their symptoms. The finding of IL-10 in some of the IL-12-positive samples probably indicates activation of the immune regulatory mechanism necessary to limit the extent of the proinflammatory response. IL-12 has been shown to induce IL-10 production. ${ }^{13}$ Candida proliferation might have stimulated production of IL-12 and IL-10 in the women positive for this organism, but this was clearly not the case for the majority of patients examined. Although women with bacterial vaginosis were excluded from this study, quantitative mi- 
crobial cultures for aerobic and anaerobic microorganisms were not available for the present subjects, so the possible role of other microbes in eliciting the vaginal symptoms remains undetermined.

The absence of vaginal IL-12 in 10 of 19 subjects who were positive for $C$. albicans strongly suggests a deficiency in the ability to mount a vaginal cell-mediated immune reaction in response to this microorganism. This lack of an appropriate immune response may contribute to susceptibility to recurrent vaginal candidiasis in these women. Similarly, detection of IL-10 in the absence of IL-12 in six women is consistent with induction of an allergic Th2-mediated immune response being involved in the symptomatology of vulvovaginitis.

The absence of IL-10, IL-12, and Candida in $32.8 \%$ of the symptomatic patients indicates the probable absence of an infectious or allergic etiology in these women. The cause(s) of their vaginal symptoms remains unknown and is currently under investigation. More detailed microbiological, immunological, and genetic testing may be necessary to resolve this question.

The absence of Candida species in most of the study subjects with symptomatic recurrent vulvoginitis points out that treatment with an antiyeast medication will be both ineffective and inappropriate for many women with recurrent vulvovaginitis. Clearly, there are multiple etiologies contributing to similar symptoms in women with vulvovaginitis and this problem is not as simple to diagnose accurately as many clinicians and lay people believe. An improved capacity to make individualized diagnoses, perhaps by incorporating testing for IL-12, IL-10, and/or other cytokines or mediators indicative of infection, allergy, or other immune processes, and subsequent appropriate treatment, may improve our capabilities to treat more effectively vulvovaginitis in these women. Further studies are needed to validate cytokine analyses for determining the etiology of vulvovaginal symptoms.

\section{REFERENCES}

1. Witkin SS. Immunologic factors influencing susceptibility to recurrent candidal vulvovaginitis. Clin Obstet Gynecol 1991;34:662-668.

2. Witkin SS, Jeremias J, Ledger WJ. A localized vaginal allergic response in women with recurrent vaginitis. J Allerg Clin Immunol 1988;81:412-416.

3. Witkin SS, Jeremias J, Ledger WJ. Recurrent vaginitis as a result of sexual transmission of $\mathrm{IgE}$ antibodies. Am J Obstet Gynecol 1988;159:32-36.

4. Witkin SS, Jeremias J, Ledger WJ. Vaginal eosinophils and $\operatorname{IgE}$ antibodies to Candida albicans in women with recurrent vaginitis. J Med Vet Mycol 1989;27:57-58.

5. Witkin SS, Hirsch J, Ledger WJ. A macrophage defect in women with recurrent Candida vaginitis and its reversal in vitro by prostaglandin inhibitors. Am J Obstet Gynecol 1986;155:790-795.

6. Rogge L, Barberis-Maino L, Biffi M, et al. Selective expression of an interleukin-12 receptor component by human T helper 1 cells. J Exp Med 1997;185:825-831.

7. Szabo SJ, Dighe AS, Gubler U, Murphy KM. Regulation of the interleukin-12R $\beta 2$ subunit expression in developing T helper 1 and Th2 cells. J Exp Med 1997;185: 817-824.

8. Kalo-Klein A, Witkin SS. Postaglandin $\mathrm{E}_{2}$ enhances and gamma interferon inhibits germ tube formation in Candida albicans. Infect Immun 1990;58:260-262.

9. Crampin AC, Mathews RC. Application of the polymerase chain reaction to the diagnosis of candidosis by amplification of an HSP 90 gene fragment. J Med Microbiol 1993;39:233-238.

10. Ledger WJ, Polaneczky MM, Yih MC, Jeremias J, Tolbert V, Witkin SS. Difficulties in the diagnosis of Candida vaginitis. Infect Dis Clin Pract 2000;9:66-69.

11. Giraldo P, Von Nowaskonski A, Gomes FA, Linhares I, Neves NA, Witkin SS. Vaginal colonization by Candida in asymptomatic women with and without a history of recurrent vulvovaginal candidiasis. Obstet Gynecol 2000;95:413-416.

12. Morace G, Sanguinetti M, Posteraro B, Lo Cascio G, Fadda G. Identification of various medically important Candida species in clinical specimens by PCRrestriction enzyme analysis. J Clin Microbiol 1997;35: 667-672.

13. Jeannin P, Delneste Y, Seveso M, Life P, Bonnefoy JY. IL-12 synergizes with IL-2 and other stimuli in inducing IL-10 production by human $\mathrm{T}$ cells. J Immunol 1996;156:3159-3165. 


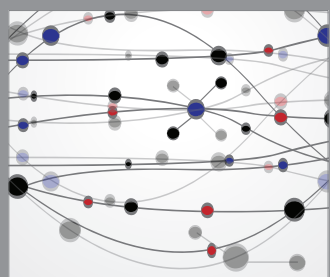

The Scientific World Journal
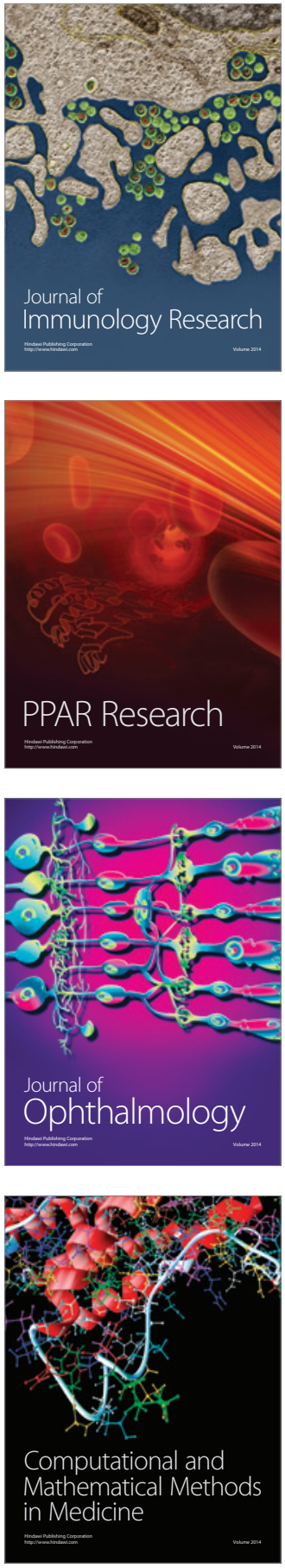

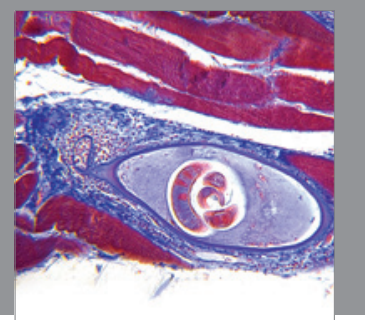

Gastroenterology

Research and Practice
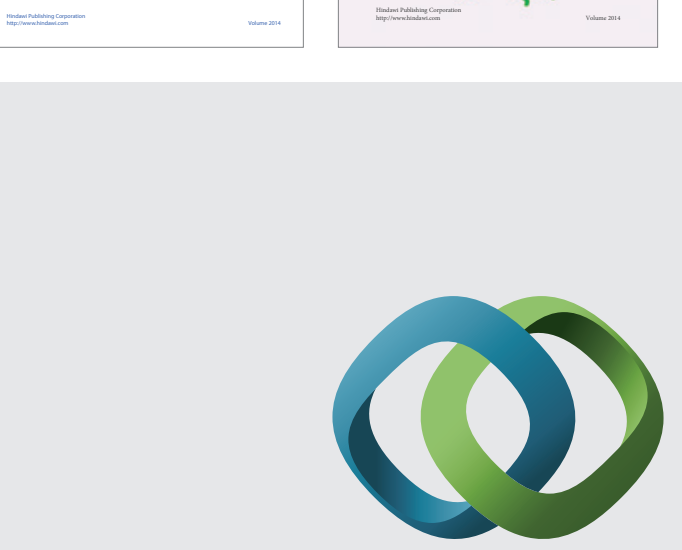

\section{Hindawi}

Submit your manuscripts at

http://www.hindawi.com
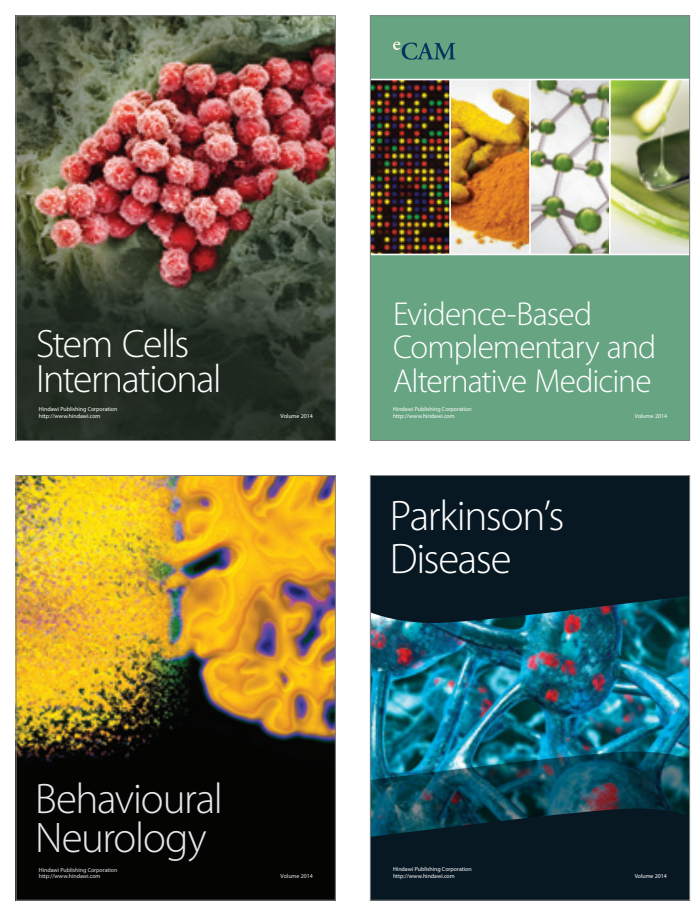

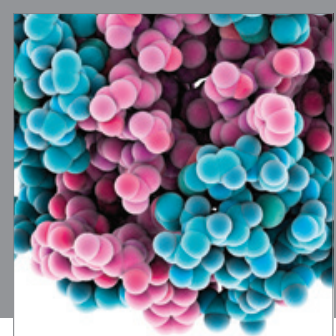

Journal of
Diabetes Research

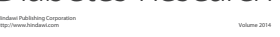

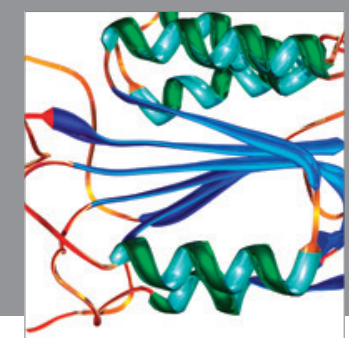

Disease Markers
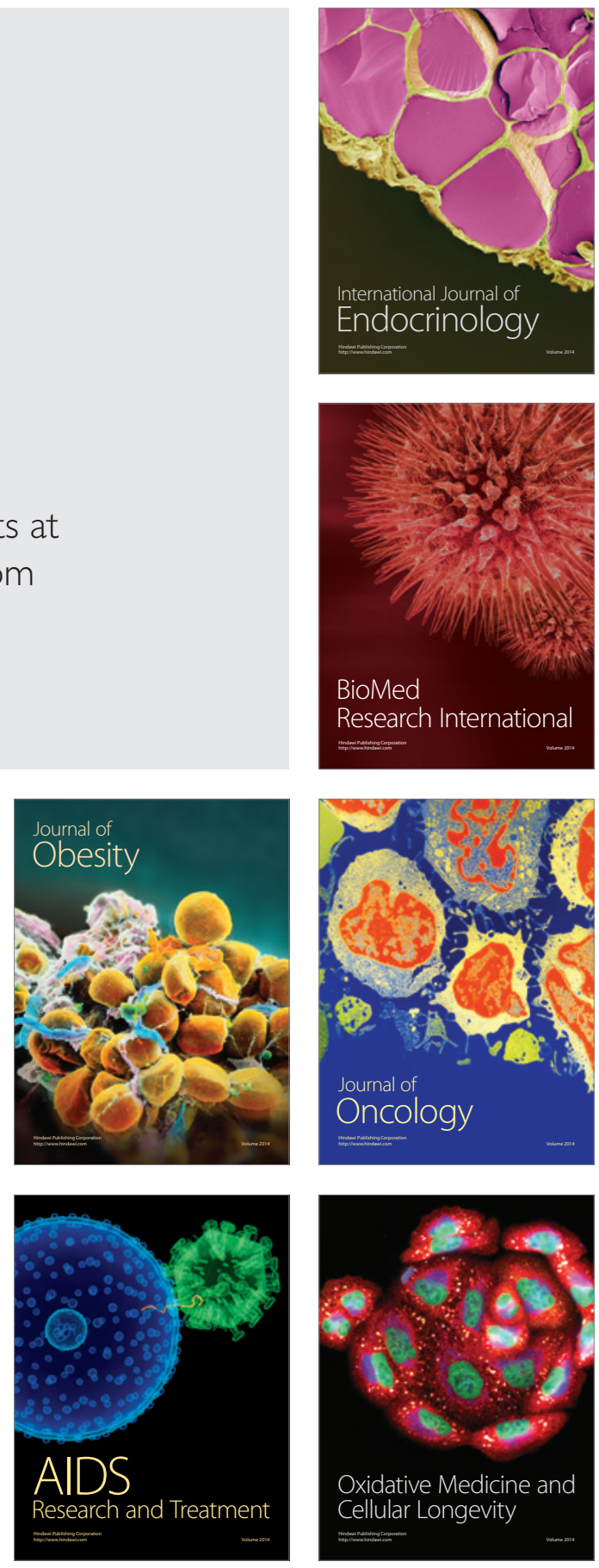